\title{
IMPACTO DA INTRODUÇÃO DE MARKET MAKERS NAS NEGOCIAÇÕES DAS BRAZILIAN DEPOSITARY RECEIPTS
}

\section{THE IMPACT OF THE INTRODUCTION OF MARKET MAKERS ON THE NEGOTIATIONS OF THE BRAZILIAN DEPOSITARY RECEIPTS}

\author{
JOÃO EDUARDO RIBEIRO \\ Universidade Federal de Minas Gerais. Endereço: Avenida Presidente Antônio \\ Carlos, 6627 - Sala 4012 | Pampulha | 31270-901 | Belo Horizonte/MG | Brasil. \\ (1) http://orcid.org/0000-0001-6969-6972 \\ joaoribeiro.cco@gmail.com
}

\author{
ANTÔNIO ARTUR DE SOUZA \\ Universidade Federal de Minas Gerais. Endereço: Avenida Presidente Antônio \\ Carlos, 6627 - Sala 4012 | Pampulha | 31270-901 | Belo Horizonte/MG | Brasil. \\ (D)http://orcid.org/0000-0002-4725-0758 \\ artur@face.ufmg.br
}

\section{GABRIEL AUGUSTO DE CARVALHO \\ Centro Federal de Educação Tecnológica de Minas Gerais. Endereço: Av. Amazonas, 7675 | Nova Gameleira | 30510-000 | Belo Horizonte/MG | Brasil. (1) http://orcid.org/0000-0002-3908-1552 ga09carvalho@gmail.com}

\section{HUDSON FERNANDES AMARAL}

Centro Universitário Unihorizontes. Endereço: Rua Alvarenga Peixoto, 1270 | Santo Agostinho | 30180-121 | Belo Horizonte/MG | Brasil. (D) http://orcid.org/0000-0001-8455-0285

hfamaral.cepead@gmail.com

\section{RESUMO}

O presente estudo se propõe a analisar o efeito gerado na liquidez das Brazilian Depositary Receipts (BDRs) com a introdução de market makers para esses ativos. A amostra desse estudo foi composta pelas BDRs com pelo menos 200 dias de negociações anteriores e posteriores à contratação dos market makers, chegando-se assim ao número final de 42 BDRs entre os anos de 2010 e 2017. Para os ativos da amostra foram obtidas as séries históricas das proxies de liquidez (bid-ask-spread, número de negócios e volume), posteriormente foi estimado um modelo AR(1) para cada BDR e então empregado o teste de Chow para se verificar a estabilidade dos parâmetros dos modelos. Os resultados encontrados na análise do teste de Chow apontaram que, para um nível de confiança de 99\%, 29 BDRs, ou seja, 69\% das BDRs estudadas, tiveram mudanças abruptas e estatisticamente significativas no bid-ask-spread, 23 BDRs $(55 \%)$ apresentaram mudanças significativas no número de negócios e 22 BDRs (52\%) apresentaram mudanças significativas no volume. Ao flexibilizar o nível de confiança para 95\%, 32 BDRs $(76 \%)$ apresentaram mudanças no bid-ask-spread, 29 BDRs (69\%) apresentaram mudanças no número de negócios e 29 BDRs, ou seja, 69\% tiveram mudanças estatisticamente significativas no volume. Esse resultado 
apresenta, portanto, fortes evidências sobre a influência dos market makers na liquidez de mercado das BDRs, mostrando que a contratação de formadores de mercado pode aumentar a liquidez e contribuir de maneira significativa com as negociações desses papéis.

Palavras-chave: Market makers. Liquidez. Teste de Chow. Brazilian Depositary Receipts.

\begin{abstract}
The present study aims to analyze the effect generated in the liquidity of the Brazilian Depositary Receipts (BDRs) with the introduction of market makers for these stocks. The sample of this study was composed by the BDRs with at least 200 days of negotiations before and after the hiring of the market makers, thus reaching the final number of 42 BDRs, between the years of 2010 and 2017. For the stocks of the sample were obtained the historical series of liquidity proxies, bid-askspread, number of trades and volume, an AR (1) model was then estimated for each BDR, and then the Chow test was used to test the stability of the model's parameters. The results found in the Chow test showed that, for a 99\% confidence level, 29 BDRs, that is, 69\% of the BDRs studied, had abrupt and statistically significant changes in bid-ask-spread, 23 BDRs (55\%) showed significant changes in the number of deals and 22 BDRs (52\%) showed significant changes in volume. When the confidence level was relaxed to 95\%, $32 \mathrm{BDRs}(76 \%)$ presented changes in the bid-ask-spread, 29 BDRs (69\%) had changes in the number of deals and 29 BDRs, that is, 69\% had statistically significant changes in volume. This result presents, therefore, strong evidence on the influence of market makers on the market liquidity of the BDRs, showing that the hiring of market makers can increase liquidity and contribute significantly to the negotiations of these papers.
\end{abstract}

Keywords: Market Makers. Liquidity. Chow's Test. Brazilian Depositary Receipts.

\title{
1 INTRODUÇÃO
}

Após as bases da Moderna Teoria de Finanças serem criadas por Markowitz (1952) e depois do surgimento dos modelos de apreçamento de ativos, como o Capital Asset Price Model (CAPM), o Consumption Capital Asset Price Model (CCAPM) e o Arbitrage Pricing Theory (APT), entre outros, abriu-se espaço para avaliar os problemas relacionados ao processo de determinação do equilíbrio de mercado. Radner (1979) argumenta que é necessário fazer uma análise mais detalhada da situação de equilíbrio, que necessita de especificações mais completas sobre os mecanismos de negociação do que as usualmente empregadas.

Nesse contexto, surge a teoria de microestrutura de mercado, que busca explicar como os diferentes mecanismos de negociação afetam os preços dos ativos e trata em detalhes as modalidades de funcionamento das negociações em um mercado, como o impacto das regras e dos modos de funcionamento dos mercados sobre as modalidades de negociação. Essa teoria abarca o os processos e resultados das trocas de ativos sob um conjunto específico de regras. O estudo da influência dos mecanismos de negociação na determinação dos preços de equilíbrio procura analisar como os mecanismos específicos de negociação, como custos de transação, assimetria informacional e liquidez de mercado, afetam o processo de formação de preços (O’Hara, 1995).

Demsetz (1968), ao tratar do efeito dos custos de transação na determinação dos preços de valores mobiliários, afirma que é possível a existência de desequilíbrios entre oferta e demanda provocados pelo efeito da dimensão tempo. No entanto, esses desequilíbrios podem ser eliminados se existirem agentes dispostos a pagar um preço pelo imediatismo. A obtenção desse preço faz que existam dois preços de equilíbrio para um mesmo mercado. Esse custo de imediatismo viabiliza um estudo mais formal sob a perspectiva da teoria de microestrutura de mercado. Como base nisso, Garman (1976) estudou como os preços das ações são determinados, dada a presença de um market 
maker, que se caracteriza como um grande participante do mercado, comprometido a manter ofertas de compra e venda de maneira regular e contínua durante a seção de negociação e negocia com incerteza de preços e estoques. O intuito do market maker é obter preços para os ativos com intenção de evitar a própria falência. Para tanto, esse agente atribui um preço mais baixo quando compra ações e um preço mais elevado quando vende, resultando num spread que o protege de uma condição de falência certa.

Para Copeland e Galai (1983), o aspecto informacional entre os participantes do mercado explica a determinação do spread. Segundo os autores, o market maker negocia com traders informados e não informados, e a determinação do spread lhe permite, com os ganhos realizados ao negociar com traders não informados, compensar as perdas ocorridas com os traders informados, mantendo-se, dessa forma, solvente. Tendo como base um cenário com assimetria informacional e atuando de maneira competitiva, os market makers promovem maior estabilidade ao mercado (Glosten, 1989).

Os market makers se inserem no contexto dos benefícios gerados pela maior liquidez dos ativos nos mercados financeiros. Diversos mercados, como a National Association of Securities Dealers Automated Quotations (Nasdaq), a Euronext e a Bolsa de Nova York (Nyse), utilizam a figura do market maker em sua estrutura de negociação. No Brasil, essa função foi regulamentada em 2003 com a Instrução CVM n. ${ }^{\circ}$ 384, e, conforme aponta Perlin (2013), o seu uso consiste em uma estratégia para promover as negociações dos ativos listados na Brasil Bolsa Balcão (B3).

A atuação desse agente no mercado brasileiro, conforme descrito por Ambrozini, Gaio, Bonacim e Cicconi (2009), visa a atração de novos investidores, o aumento da base de acionistas do ativo e a elevação direta da liquidez. A função de market maker, conforme definido pela legislação vigente, pode ser exercida por traders especializados, como distribuidoras e corretoras de valores mobiliários, bancos múltiplos e bancos de investimentos. Além disso, destaca-se que o market maker atua de maneira diferente de um investidor comum, uma vez que seu interesse não reside na especulação financeira ou na imunização de carteiras, mas na compensação recebida na forma do spread pelo provimento de liquidez para o mercado. O spread gerado é maior para ativos de maior volatilidade nos preços, dado o maior risco de inventário gerado pela manutenção desse ativo na carteira pelo market maker (Perlin, 2013).

Perlin (2013) chegou às mesmas conclusões de Ambrozini et al. (2009), que indica que a introdução de market makers é vista pelo mercado como um evento positivo para o futuro das empresas negociadas na B3, pois parece afetar a liquidez e outras variáveis relacionadas aos ativos financeiros. Além de empresas brasileiras, a B3 negocia também as chamadas Brazilian Depositary Receipts (BDRs) - valores mobiliários emitidos no Brasil e que possuem como lastro ativos, geralmente ações, emitidos no exterior. As BDRs são um mecanismo ideal para empresas estrangeiras se estabelecerem no mercado de capitais brasileiro. Como motivos que levam as empresas estrangeiras a acessarem o Mercado Internacional, listando um programa de BDR, podese citar maior visibilidade entre consumidores, diversificação e ampliação de sua base de acionistas, clientes e investidores, melhor valuation internacional ao se colocar junto a seus pares, aumento da liquidez total de sua ação ao atrair novos investidores, entre outros. Além disso, operar no Brasil contribui para futuras ofertas públicas, aquisições e consolidação do crescimento empresarial (B3, 2017).

Em vista disso, o presente estudo se propõe a responder ao seguinte questionamento: Qual o efeito da introdução dos market makers no aumento da liquidez das BDRs? Além da importância das BDRs para o mercado de capitais brasileiro e da relevância de se entender o impacto dos market makers nesse instrumento financeiro, há também o interesse acadêmico no intuito de observar o comportamento da liquidez e inferir relações de causalidade nesses mercados, como os encontrados em estudos de Banz (1981), Morais e Portugal (1999), Pereira (2006), Neves (2007), Correia, Amaral e Bressan (2008), entre outros. Dessa forma, é importante que a academia continue contribuindo com estudos que ajudem a compreender o ofício do mercado de capitais brasileiro. 
Para uma melhor compreensão do conteúdo a ser apresentado, além desta introdução, este estudo é composto ainda por outras 4 seções. A Seção 2 traz uma breve fundamentação teórica sobre a teoria de microestrutura de mercado, a liquidez de mercado e a atuação dos market makers. A Seção 3 descreve a metodologia utilizada neste estudo, a amostra, as variáveis e o modelo econométrico empregado. Na Seção 4 é exposta a análise dos resultados encontrados, e, por fim, a Seção 5 traz as conclusões do estudo.

\section{FUNDAMENTAÇÃO TEÓRICA}

No tocante ao desenvolvimento e compreensão dos aspectos abordados neste estudo, é ponto substancial a definição de microestrutura de mercado, em que se destaca O'Hara (1995) que aponta a microestrutura como alusiva ao estudo de determinado mercado sob regras explícitas, envolvendo desde o processo de negociação até os atributos que impactam na precificação dos ativos. Nessa perspectiva, Madhavan (2000) ressalta que a teoria de microestrutura de mercado devota-se em estudar como as demandas dos investidores impactam nos preços dos ativos e no desenvolvimento do mercado. Dentre os diversos estudos que abarcam a microestrutura de mercado, evidenciam-se Stoll (1978), Kyle (1985), Glosten e Milgrom (1985), Amihud e Mendelson (1987), O'Hara (1995) e Madhavan (2000).

Em Stoll (1978), um dos trabalhos precursores sobre microestrutura, a figura do market maker desempenha um papel crucial na formação do preço dos ativos, atribuindo o spread aos custos de estoque incorporados no intuito de promover liquidez ao mercado. Por outro lado, Amihud e Mendelson (1987) estudaram os impactos do mecanismo de negociação e o comportamento de preços nas ações da Nyse e entenderam que o mecanismo de negociação tem um efeito significativo no comportamento do preço das ações. Alternativamente a Stoll (1978), o entendimento de Glosten e Milgrom (1985) é de que o spread pode refletir a assimetria de informação existente entre traders informados e não informados. Kyle (1985), por sua vez, sugeriu um modelo no qual há três tipos de agentes no mercado: os irracionais (noise traders), os informados (insider, neutro ao risco) e os market makers, que observam as atividades dos demais agentes e alteram os preços dos ativos.

O'Hara (1995) fortaleceu a teoria da microestrutura de mercado. A autora ressalta em seu estudo que os preços dos ativos se ajustam a novas informações e que em um mundo perfeito, as novas informações seriam instantaneamente difundidas e analisadas por todos os traders do mercado, que rapidamente ajustariam o preço dos ativos amparados nas preferencias dos agentes. Esse mundo perfeito em que os preços refletem completamente todas as informações disponíveis no mercado é denominado hipótese do mercado eficiente (HME) por Fama (1970, 1991). No entanto, para O'Hara (1995), a teoria de microestrutura de mercado flexibiliza os pressupostos do HME e se concentra nos estudos de formação de preços, na análise de risco, nos modelos de transparência das negociações, no desenho de protocolos e regras de mercado e na liquidez dos ativos. Em outras palavras, a teoria da microestrutura de mercado abarca toda a estrutura informacional e institucional nas quais as transações financeiras são efetuadas.

O’Hara (1995) propôs modelos orientados para a compreensão do problema do market maker, que por estar sempre presente em negociações de compra e venda de ativos, e com o objetivo de manter a solvência e maximizar os ganhos, estabelece o preço de negociação. Por desejar manter uma carteira eficiente e por necessidade de compensar o risco de carregar uma carteira não eficiente, o market maker define preços diferentes de compra e venda, gerando, dessa forma, o spread. Para O'Hara (1995), essa atuação dos market makers interfere na liquidez dos ativos e é denominada problema de seleção adversa.

Por fim, Madhavan (2000) investigou a literatura empírica e teórica a respeito da microestrutura de mercado; envolvendo a formação de preços, incluindo o processo dinâmico pelo qual os preços incorporam informações; a capacidade dos participantes do mercado de observar informações sobre o processo de negociação; a estrutura e o design do mercado, incluindo a relação 
entre a formação de preços e os protocolos de negociação; e as aplicações para outras áreas de participação, incluindo nesse caso os preços de ativos, investimentos internacionais e empresas. Para o autor, a microestrutura de mercado estuda o processo pelo qual as demandas latentes dos investidores são traduzidas em preços e volumes.

Nessa perspectiva, a liquidez de mercado desempenha um papel indispensável na demanda dos investidores que desejem concentrar seus investimentos em portfólios que podem ser rapidamente negociados com baixo custo de transação. Amihud e Mendelson (1986) definem a liquidez de mercado como o custo da imediata execução de uma ordem de compra ou venda e dizem que ela está relacionada ao risco na precificação dos ativos financeiros e que, em condições de equilíbrio, o retorno dos ativos deve ser determinado em uma função decrescente da liquidez, isto é, para os autores, há um prêmio de liquidez na precificação de ativos.

Em um estudo mais recente, Amihud e Mendelson (2008) ressaltam que a liquidez de um ativo impacta também a estrutura de capital da empresa, dado que, caso as ações e os títulos negociados por uma empresa sejam de menor liquidez, o retorno exigido pelos investidores será maior. Os autores concluem ainda que medidas que aumentem a liquidez dos ativos são benéficas para as empresas, como, por exemplo, a publicidade em torno da empresa, o nível de informações divulgadas, o tamanho do lote de negociação dos ativos, e também a contratação de um market maker.

Por causa disso, no intuito de aumentar a liquidez, atrair mais investidores e pulverizar a base de acionistas, as companhias optam por fazer uso da figura do market maker. A promoção de liquidez por esses agentes se dá de forma complexa, pois os traders podem ter informações privadas a respeito de um ativo e os market makers não. Nos mercados de alta frequência, os market makers buscam obter pequenos ganhos que se expandem em transações com grandes quantidades de ordens, e seus ganhos dependem exclusivamente do controle do risco de ser alvo de seleção adversa. A probabilidade de ganho desses market makers, ao transacionarem grandes quantidades de ativos, aumenta quando há equilíbrio entre os fluxos de ordens de compra e venda. Quando não existe esse equilíbrio há chances de os market makers serem alvos de seleção adversa e, consequentemente, devido à alta toxicidade, eles liquidarem suas posições, diminuindo a liquidez do mercado (Siqueira, Amaral \& Correia, 2017).

Por causa da relação entre liquidez de mercado e retorno dos ativos, torna-se importante compreender como os agentes impactam a liquidez dos mercados. Venkataraman e Waisburd (2007), ao analisarem a reação do mercado acionário francês ao anúncio da entrada de market makers nas negociações, concluíram que o mercado reage positivamente ao anúncio do uso de market makers, com uma variação positiva e significativa na liquidez e no retorno das ações, logo após o anúncio. No mesmo entendimento de Venkataraman e Waisburd (2007), Perotti e Rindi (2010) investigaram o efeito da entrada de market makers nas ações da bolsa de valores italiana e concluíram que a entrada desses agentes de liquidez aumentou o número de negócios e diminuiu os spreads e a volatilidade dos preços. Clark-Joseph, Ye e Zi (2017) estudaram o impacto dos market makers na liquidez das ações americanas. Para os autores, os market makers melhoram significativamente a liquidez no mercado eletrônico moderno.

No Brasil, Ambrozini et al. (2009) estudaram, no período de março de 2003 a dezembro de 2007, a relação entre a atuação dos market makers e o aumento da liquidez das ações das companhias brasileiras de capital aberto. Esses autores concluíram que há evidências significativas de que a contratação de market makers pode aumentar a liquidez das ações brasileiras negociadas na B3, facilitando as negociações desses títulos no mercado. Assim como Ambrozini et al. (2009), Costa e Salles (2010) verificaram, a partir de séries temporais, a influência da adoção de market makers pelas empresas brasileiras na liquidez de suas ações. Para tanto, os autores selecionaram uma amostra de 10 ações com negociações de janeiro a maio de 2009 e determinaram séries temporais usando modelos heteroscedásticos de volatilidade para determinação da liquidez. Os resultados apontaram que os benefícios da adoção do formador de mercado foram válidos apenas 
para parte dos ativos analisados e que seus benefícios não se propagaram na mesma intensidade por todas as empresas que utilizaram formadores de mercado.

Sanvitto (2011) partiu de uma amostra maior que a utilizada por Costa e Salles (2010) e testou os resultados da atuação de market makers nas ações das empresas que optaram pela contratação do serviço a partir de 2003. Os resultados do estudo apontam que a atuação dos market makers proveu liquidez e impactou positivamente o valor de mercado das empresas contratantes. Silva (2012), por sua vez, analisou 204 empresas negociadas na B3 e que contrataram market makers, a fim de verificar se essas empresas obtiveram aumento muito significativo na quantidade de negócios, no volume negociado e, por consequência, no aumento de liquidez de suas ações. $\mathrm{O}$ autor identificou diferenças positivas ocorridas após a contratação dos market makers em todas as variáveis analisadas, principalmente na quantidade de negócios e no volume negociado, demonstrando que a atuação dos market makers elevou a liquidez das ações analisadas.

Rogers e Mamede (2014) fizeram dois estudos de eventos em 66 empresas que contratam market makers no período de janeiro de 2013 a dezembro de 2014. O primeiro evento com a data de divulgação da contratação do market maker e o segundo com a data de começo de atuação. Os resultados apontam que entre 15 dias antes e após a contratação dos market makers houve presença de retornos anormais positivos, o que para os autores gera subsídios para discutir a precificação da liquidez, que até a data de realização do estudo não foram encontradas no mercado de capitais brasileiro.

Há, como visto, diversos estudos demostrando que a atuação dos market makers tem apresentado um impacto positivo na liquidez de diversos mercados. No entanto, não foram encontrados estudos sobre a atuação desse agente na liquidez das BDRs. Isso evidencia a necessidade de pesquisas que busquem preencher essa lacuna.

\section{PROCEDIMENTOS METODOLÓGICOS}

Este trabalho é de carácter descritivo e de abordagem quantitativa, que para Martins e Theóphilo (2007) são estudos que visam fazer uma análise ou descrição dos dados por meio de métodos estatísticos. Os dados, secundários, foram obtidos no sítio eletrônico da B3 e no sistema de informações da Bloomberg e tratados pelo programa Excel® 2016. Posteriormente, para realização do método estatístico, foi utilizado o software Gretl 2018®.

Dos 255 ativos negociados por market makers na data de coleta dos dados (30/11/2017), 5 eram Unit (Certificado de Depósito de Ações), 4 FII (Fundo de Investimento Imobiliário), 15 ETF (Exchange Traded Funds), 127 BDRs e 104 ações. O universo de dados para o estudo é composto pelas 127 BDRs. Todavia, para a realização do teste de quebra estrutural de Chow, a ser empregado neste estudo, é necessário que tenha havido negociação das BDRs antes da contratação dos market makers. Por isso, foi necessário verificar as datas de contratação desses formadores de mercado e a data da primeira negociação que o sistema de informações da Bloomberg forneceu sobre cada BDR.

Após essa verificação e exclusão das BDRs que não se encaixavam nos critérios de análise, chegou-se a um número final de 42 BDRs com pelo menos 200 dias de negociações anteriores à contratação dos market makers. A escolha dessas 42 BDRs se deu no intuito de evitar conclusões errôneas causadas pela volatilidade do mercado brasileiro. O período de tempo escolhido compreende de 29/11/2010 (primeiro dia de vigência de atuação de um market maker em BDR) até 31/12/2017. Buscou-se, com isso, trabalhar com o maior intervalo de tempo possível. A Tabela 1 apresenta uma relação com as 42 BDRs presentes na amostra, o market maker contratado e a data da primeira atuação do market maker. 
Tabela 1

Amostra da pesquisa

\begin{tabular}{|c|c|c|c|c|c|}
\hline Tickers & Market maker & Início de atuação & Tickers & Market maker & Início de atuação \\
\hline AALC34 & Credit Suisse & $01 / 09 / 2016$ & DISB34 & Bradesco & $21 / 09 / 2015$ \\
\hline BLAK34 & Credit Suisse & $18 / 05 / 2017$ & DUPO34 & Credit Suisse & $18 / 05 / 2016$ \\
\hline BOAC34 & Guide Invest. & 04/07/2016 & EXXO34 & Guide Invest. & 04/07/2016 \\
\hline COCA34 & Guide Invest. & $11 / 08 / 2014$ & FCXO34 & Guide Invest. & $27 / 09 / 2013$ \\
\hline GPIV33 & Credit Suisse & $21 / 03 / 2013$ & FDMO34 & Guide Invest. & $27 / 09 / 2013$ \\
\hline MCDC34 & Guide Invest. & 04/07/2016 & GEOO34 & Guide Invest. & $27 / 09 / 2013$ \\
\hline MRCK34 & Guide Invest. & $27 / 09 / 2013$ & GSGI34 & Guide Invest. & 04/07/2016 \\
\hline ORCL34 & Guide Invest. & $11 / 08 / 2014$ & HALI34 & Guide Invest. & $27 / 09 / 2013$ \\
\hline SLBG34 & Guide Invest. & $11 / 08 / 2014$ & ITLC34 & Guide Invest. & $27 / 09 / 2013$ \\
\hline USSX34 & Credit Suisse & $01 / 09 / 2016$ & MSCD34 & Guide Invest. & $11 / 08 / 2014$ \\
\hline WFCO34 & Guide Invest. & $27 / 09 / 2013$ & MSFT34 & Guide Invest. & $27 / 09 / 2013$ \\
\hline AAPL34 & Guide Invest. & 04/07/2016 & NFLX34 & Guide Invest. & $27 / 09 / 2013$ \\
\hline AMGN34 & Guide Invest. & $27 / 09 / 2013$ & NIKE34 & Guide Invest. & $11 / 08 / 2014$ \\
\hline AMZO34 & Guide Invest. & $11 / 08 / 2014$ & PFIZ34 & Guide Invest. & 04/07/2016 \\
\hline ARMT34 & Guide Invest. & 04/07/2016 & PGCO34 & Guide Invest. & $27 / 09 / 2013$ \\
\hline AVON34 & Guide Invest. & $04 / 07 / 2016$ & QCOM34 & Guide Invest. & $27 / 09 / 2013$ \\
\hline CATP34 & Guide Invest. & $11 / 08 / 2014$ & TIFF34 & Guide Invest. & $22 / 02 / 2016$ \\
\hline CHVX34 & Guide Invest. & $11 / 08 / 2014$ & TWTR34 & Credit Suisse & $14 / 12 / 2015$ \\
\hline CMCS34 & Guide Invest. & $27 / 09 / 2013$ & USBC34 & Guide Invest. & $22 / 02 / 2016$ \\
\hline COLG34 & Guide Invest. & $11 / 08 / 2014$ & VERZ34 & Guide Invest. & $27 / 09 / 2013$ \\
\hline CSCO34 & Guide Invest. & $27 / 09 / 2013$ & WALM34 & Guide Invest. & 04/07/2016 \\
\hline
\end{tabular}

Fonte: Adaptado de Brasil Bolsa Balcão (B3). BDRs - Brazilian Depositary Receipts. Recuperado em 11 dezembro, 2017, de http://www.bmfbovespa.com.br/pt_br/listagem/bdrs-brazilian-depositary-receipts/

No tocante às medidas de liquidez, segundo Machado e Medeiros (2011), não há uma medida plenamente aceita na literatura que capture todas as dimensões da liquidez. Com isso, na intenção de captar as múltiplas dimensões da liquidez de mercado, optou-se pela utilização de três proxies: o bid-ask-spread, o número de negócios e o volume de negociações. O bid-ask-spread (Equação 1) é a diferença entre o maior preço de compra (bid) e o menor preço de venda (ask) do ativo no mercado. Proposto por Demsetz (1968), o autor sugere que quanto maior o bid-askspread, menor a liquidez do ativo em questão. Essa proxy já foi utilizada por Amihud e Mendelson (1986), Amihud e Mendelson (1991), entre outros. A segunda proxy analisada, número de negócios, consiste na quantidade de negócios realizados com o ativo e foi utilizada em estudos como Correia et al. (2008), Vieira e Milach (2008) e Machado e Medeiros (2011). Por fim, a terceira proxy, volume de negociações, representa o volume de negociações diárias de cada ativo e foi utilizada em estudos como Chordia, Subrahmanyam e Anshuman (2001), Jun, Marathe e Shawky (2003) e Correia et al. (2008).

$$
\operatorname{Spread}_{t}=\operatorname{ask}_{t}-\text { bid }_{t}
$$

Em que:

Spread $_{t}$ é a diferença entre a oferta de venda e a oferta de compra no tempo $t$;

$a s k_{t}$ é a oferta de venda no tempo $t$; e

$b i d_{t}$ é a oferta de compra no tempo $t$.

Para a realização da análise das séries temporais das BDRs que contrataram market makers foi aplicada a metodologia estatística de teste de quebra estrutural proposta por Chow (1960), cujo intuito é verificar se houve alguma mudança de comportamento (mudanças abruptas) na série 
temporal num dado instante de tempo. Aplicou-se, dessa forma, o teste de Chow nas séries de bidask-spread, número de negócios e volume de cada uma das BDRs, a fim de descobrir se existiu alguma mudança nessas proxies no período posterior à contratação dos market makers. A análise do teste de Chow foi realizada com base no seu p-valor ( $p$-value), de interpretação direta, isto é, a probabilidade de rejeitar a hipótese nula de que a série não apresenta quebra estrutural. Os níveis de confiança foram firmados em $90 \%, 95 \%$ e $99 \%$.

A escolha do teste de Chow como ferramenta de análise se deu em função do teste ser considerado um dos principais e mais eficientes testes de quebra estrutural na literatura econométrica. Além do mais, a partir do teste de Chow, pode-se determinar e testar a quebra, dado um ponto específico na série que se suspeite acontecer. É um teste que necessita de uma especificação, a priori, de quando ocorrerá uma quebra estrutural (Ambrozini et al., 2009). Optouse por utilizar o modelo de série temporal autorregressivo de primeira ordem (AR(1)) proposto por Box e Jenkins (1970) e com estrutura similar a apresentada na Equação 1, uma vez que o objetivo é analisar uma série contendo apenas uma variável.

$$
Y_{t}=\alpha+\beta Y_{t-1}+\varepsilon_{t}
$$

Em que:

$\alpha$ e $\beta$ são constantes desconhecidas e $-1<\beta<1$;

$\varepsilon_{t}$ é um erro aleatório, normalmente distribuído com média zero e desvio padrão $\sigma$.

As séries temporais de cada proxy foram divididas em três amostras. A amostra I foi composta por dados anteriores à contratação dos market makers, a amostra II foi composta por dados posteriores à contratação e a amostra III foi formada por todos os dados das proxies das 42 BDRs estudadas. Para que fique demostrado que a atuação dos market makers impactaram na liquidez dos ativos é preciso que haja diferenças estatisticamente significativas entre a soma dos quadrados dos resíduos (SQR) da amostra III com a SQR das amostras I e II.

Após o resultado, rejeita-se ou não a hipótese nula com base no p-valor e nos níveis de significância de $1 \%, 5 \%$ e $10 \%$. A análise do p-valor, também conhecido como probabilidade de significância ou nível descritivo, representa a probabilidade de se obter uma estatística de teste igual ou mais extrema que a observada em uma amostra, sob a hipótese nula. Nos testes de hipóteses, o resultado é estatisticamente significante quando o p-valor observado é menor que o nível de significância $\alpha$ estabelecido. A hipótese nula $(\mathrm{H} 0)$ utilizada na realização do teste de Chow foi a de que os parâmetros das regressões são iguais para as diferentes subamostras; isto é, não existe quebra estrutural. Ao rejeitar a hipótese nula indica-se que a contratação de market makers surtiu efeito sobre as proxies analisadas (bid-ask-spread, número de negócios e volume), resultando na alteração da liquidez de mercado das BDRs.

\section{ANÁLISE E INTERPRETAÇÃO DOS RESULTADOS}

A fim de verificar se houve impacto na liquidez das 42 BDRs após a contratação de market makers realizou-se o teste de estabilidade de Chow nas proxies bid-ask-spread, número de negócios e volume. Neste estudo foi estabelecido diferentes níveis de significância em 1\%, $5 \%$ e $10 \%$. Sempre que o p-valor é maior que o nível de significância estabelecido não é possível rejeitar H0, ou seja, não há mudança abrupta na série temporal da proxy analisada, o que sugere não haver aumento da liquidez com a contratação do market maker. Quando o contrário ocorre, rejeita-se H0, ou seja, há indicações de mudanças na liquidez da BDR após a contratação do market maker.

A Tabela 2 apresenta os valores referentes ao teste, identificados pelo seu p-valor, para as 42 BDRs analisadas. Conforme observado, dados os níveis de significância estabelecidos, não é possível rejeitar H0 na análise das BDRs BOAC34, MCDC34, MRCK34, CSCO34, GSGI34, NFLX34 e PFIZ34, ou seja, dado um nível de significância de 10\%, apenas em 7, das 42 BDRs analisadas, não se pode rejeitar H0. Nesse sentido, quando medida pela proxy bid-ask-spread, a 
contratação dos market makers parece não ter causado impacto na liquidez de mercado dessas BDRs. Aplicou-se também o teste de quebra estrutural de Chow para outra proxy de liquidez de mercado, o número de negócios. Analisando os resultados do p-valor, dado o nível de significância de 10\%, não é possível rejeitar H0 para os testes das BDRs AALC34, SLBG34, AVON34, FDMO34, GSGI34, HALI34, NFLX34, NIKE34, QCOM34 e USBC34, ou seja, a contratação dos market makers parece não ter causado impacto na liquidez de mercado nos papéis dessas BDRs quando medida pela proxy número de negócios. O resultado para essas BDRs vai ao encontro do estudo de Rogers e Mamede (2014), que verificaram um aumento na liquidez das ações que contrataram market makers no período de janeiro de 2013 a dezembro de 2014.

Por meio dos valores do teste de Chow, identificados pelo p-valor, para as 42 BDRs analisadas por meio da última proxy do estudo, o volume, não foram identificadas mudanças estruturais abruptas nos parâmetros do volume das BDRs AALC34, BOAC34, ORCL34, SLBG34, AVON34, CHVX34, DUPO34, EXXO34, FDMO34, GSGI34, TWTR34 e USBC34 a um nível de significância de 10\%, ou seja, em 12, das 42 BDRs analisadas, não foi possível rejeitar H0, indicando que a contratação dos market makers parece não ter causado impacto na liquidez dessas BDRs, quando medida pela proxy volume de negociações.

Foi possível observar que, das proxies analisadas, o bid-ask-spread teve o maior impacto com a adoção dos market makers, seguido pelo número de negócios e, por último, pelo volume. No entanto, mesmo com divergência entre as proxies de liquidez, a contratação desses formadores de mercado se mostrou uma boa alternativa para as BDRs que desejem aumentar a liquidez dos seus papéis. O resultado vai ao encontro dos resultados de Perlin (2013) para o mercado de capitais brasileiro e supera os encontrados por Ambrozini et al. (2009), em que os autores encontraram evidências de aumento na liquidez em apenas $52 \%$ das ações a um nível de significância de $10 \%$.

Tabela 2

\section{Resultado p-valor do Teste de Chow}

\begin{tabular}{cccc|cccc}
\hline Tickers & Bid-ask-spread & $\begin{array}{c}\text { Número de } \\
\text { negócios }\end{array}$ & Volume & Tickers & Bid-ask-spread & $\begin{array}{c}\text { Número de } \\
\text { negócios }\end{array}$ & Volume \\
\hline AALC34 & 0,0000 & 0,2300 & 0,2300 & DISB34 & 0,0100 & 0,0000 & 0,0000 \\
BLAK34 & 0,0000 & 0,0100 & 0,0100 & DUPO34 & 0,0000 & 0,0000 & 1,0000 \\
BOAC34 & 0,1200 & 0,0000 & 0,3500 & EXXO34 & 0,0000 & 0,1000 & 0,4400 \\
COCA34 & 0,0000 & 0,0000 & 0,0000 & FCXO34 & 0,0300 & 0,0400 & 0,0200 \\
GPIV33 & 0,0000 & 0,0000 & 0,0000 & FDMO34 & 0,0000 & 0,1500 & 0,4100 \\
MCDC34 & 0,2800 & 0,0000 & 0,0400 & GEOO34 & 0,0000 & 0,0000 & 0,0000 \\
MRCK34 & 0,1200 & 0,0000 & 0,0000 & GSGI34 & 0,2800 & 0,1700 & 0,2200 \\
ORCL34 & 0,0000 & 0,0200 & 0,5000 & HALI34 & 0,0800 & 0,3600 & 0,0200 \\
SLBG34 & 0,0000 & 0,3500 & 0,2300 & ITLC34 & 0,0000 & 0,0000 & 0,0000 \\
USSX34 & 0,0000 & 0,0100 & 0,0000 & MSCD34 & 0,0000 & 0,0000 & 0,0000 \\
WFCO34 & 0,0000 & 0,1000 & 0,0600 & MSFT34 & 0,0000 & 0,0000 & 0,0000 \\
AAPL34 & 0,0000 & 0,0000 & 0,0000 & NFLX34 & 0,2600 & 0,4000 & 0,0200 \\
AMGN34 & 0,0400 & 0,0000 & 0,0000 & NIKE34 & 0,0000 & 0,1200 & 0,0000 \\
AMZO34 & 0,0000 & 0,0100 & 0,0000 & PFIZ34 & 0,8500 & 0,0000 & 0,0000 \\
ARMT34 & 0,0000 & 0,0200 & 0,0100 & PGCO34 & 0,0900 & 0,0000 & 0,0000 \\
AVON34 & 0,0000 & 0,1200 & 0,2300 & QCOM34 & 0,0000 & 0,1900 & 0,0400 \\
CATP34 & 0,0000 & 0,0000 & 0,0000 & TIFF34 & 0,0100 & 0,0300 & 0,0300 \\
CHVX34 & 0,0000 & 0,0500 & 0,2000 & TWTR34 & 0,0600 & 0,1000 & 0,7200 \\
CMCS34 & 0,0000 & 0,0000 & 0,0000 & USBC34 & 0,0000 & 0,4600 & 0,4500 \\
COLG34 & 0,0000 & 0,0000 & 0,0000 & VERZ34 & 0,0200 & 0,0400 & 0,0200 \\
CSCO34 & 0,1200 & 0,0100 & 0,0100 & WALM34 & 0,0100 & 0,0000 & 0,0100 \\
\hline Fon & & & & & & \\
\hline
\end{tabular}

Fonte: Dados da pesquisa (2019). 
A fim de facilitar a visualização dos resultados, as tabelas 3, 4 e 5 resumem a Tabela 2, apresentando o percentual de BDRs que rejeitaram $\mathrm{H} 0$ para os níveis de significância de 1\%, 5\% e $10 \%$ para as proxies bid-ask-spread, número de negócios e volume, respectivamente. Os resultados expressos nas tabelas evidenciam o número e o percentual de BDRs, dentre as 42 analisadas, que demostraram uma possível elevação na liquidez após a contratação dos market makers.

Analisando a Tabela 3, pode-se perceber que a atuação dos market makers nas BDRs parece diminuir o bid-ask-spread delas. O resultado sugere haver um aumento na liquidez de 29 BDRs analisadas (69\%) ao nível de significância de 1\%. Ao elevarmos o nível de significância para 5\% e 10\%, nota-se um aumento na liquidez de 32 (76\%) e $35(83 \%)$ BDRs, respectivamente. Ao analisar a série temporal da proxy bid-ask-spread verificou-se, portanto, que os market makers parecem contribuir na promoção da liquidez das BDRs.

Tabela 3

Percentual de BDRs de acordo com a hipótese de existência de quebra estrutural (bid-askspread)

\begin{tabular}{cccc}
\hline & $\mathbf{1 \%}$ & $\mathbf{5 \%}$ & $\mathbf{1 0 \%}$ \\
\hline Existe quebra estrutural & $29(69 \%)$ & $32(76 \%)$ & $35(83 \%)$ \\
Não existe quebra estrutural & $13(31 \%)$ & $10(24 \%)$ & $7(17 \%)$ \\
\hline Total & $\mathbf{4 2 ( 1 0 0 \% )}$ & $\mathbf{4 2 ( 1 0 0 \% )}$ & $\mathbf{4 2 ( 1 0 0 \% )}$ \\
\hline
\end{tabular}

Fonte: Dados da pesquisa (2019).

O resultado evidenciado pela Tabela 3 vai ao encontro do estudo de Perlin (2013). Ambrozini et al. (2009), ao estudar o mercado acionário brasileiro, encontrou evidências de aumento na liquidez em apenas $52 \%$ das ações a um nível de significância de $10 \%$. O resultado do teste de Chow para o bid-ask-spread sugere, portanto, que nas BDRs a contratação dos market makers parece surtir um efeito em mais papéis do que nas ações brasileiras. Nesse sentido, o aumento na liquidez causada pela presença do market maker indica que a contratação desses formadores de mercado pode ser interessante para as BDRs, uma vez que somente em 17\% (a 10\% de significância) das BDRs analisadas parece não ter havido uma diminuição do bid-ask-spread, ou seja, um aumento da liquidez.

Tabela 4

Percentual de BDRs de acordo com a hipótese de existência de quebra estrutural (número de negócios)

\begin{tabular}{cccc}
\hline & $\mathbf{1 \%}$ & $\mathbf{5 \%}$ & $\mathbf{1 0 \%}$ \\
\hline Existe quebra estrutural & $23(55 \%)$ & $29(69 \%)$ & $32(76 \%)$ \\
Não existe quebra estrutural & $19(45 \%)$ & $13(31 \%)$ & $10(24 \%)$ \\
\hline Total & $\mathbf{4 2}(\mathbf{1 0 0 \%})$ & $\mathbf{4 2}(\mathbf{1 0 0 \%})$ & $\mathbf{4 2}(\mathbf{1 0 0 \%})$
\end{tabular}

Fonte: Dados da pesquisa (2019).

Examinando a Tabela 4, percebe-se que a atuação dos market makers nas BDRs parece aumentar o número de negócios delas. O resultado obtido pelo teste sugere um aumento na liquidez de 23 BDRs analisadas (55\%) ao nível de significância de 1\%. Ao elevarmos o nível de significância para 5\% e 10\%, nota-se um aumento no número de negócios de 29 (69\%) e 32 (76\%) BDRs respectivamente. Ao comparamos com a proxy analisada anteriormente, nota-se que a contratação de market makers pode ter diminuído o bid-ask-spread de mais BDRs do que aumentou o número de negócios. Ao nível de significância de $10 \%$, por exemplo, nota-se que $83 \%$ das BDRs analisadas tiveram diminuição do bid-ask-spread, mostrando dessa forma uma elevação 
na liquidez. Por outro lado, em $76 \%$ das BDRs em estudo, percebeu-se um aumento no número de negócios. Nota-se ainda que algumas BDRs que não sofreram mudança no bid-ask-spread sofreram mudanças no número de negócios.

Na Tabela 5 os resultados mostram que a atuação dos market makers nas BDRs parece aumentar o volume de negociações delas. $\mathrm{O}$ resultado obtido pelo teste sugere um aumento na liquidez de 22 BDRs analisadas (52\%) ao nível de significância de $1 \%$. Ao elevarmos o nível de significância para 5\% e 10\%, nota-se um aumento na liquidez de 29 (69\%) e 30 (71\%) das BDRs respectivamente.

Tabela 5

Percentual de BDRs de acordo com as hipóteses de existência de quebra estrutural (volume)

\begin{tabular}{cccc}
\hline & $\mathbf{1 \%}$ & $\mathbf{5 \%}$ & $\mathbf{1 0 \%}$ \\
\hline Existe quebra estrutural & $22(52 \%)$ & $29(69 \%)$ & $30(71 \%)$ \\
Não existe quebra estrutural & $20(48 \%)$ & $13(31 \%)$ & $12(29 \%)$ \\
\hline Total & $\mathbf{4 2}(\mathbf{1 0 0 \%})$ & $\mathbf{4 2}(\mathbf{1 0 0 \%})$ & $\mathbf{4 2}(\mathbf{1 0 0 \%})$ \\
\hline
\end{tabular}

Fonte: Dados da pesquisa (2019).

O resultado vai ao encontro dos resultados de Perlin (2013) para o mercado de capitais brasileiro e, mesmo estando abaixo das conclusões da análise das proxies bid-ask-spread e número de negócios, ainda supera os resultados de Ambrozini et al. (2009), que encontrou evidências de aumento na liquidez em apenas $52 \%$ das ações brasileiras, a um nível de significância de $10 \%$.

Na sequência, a Tabela 6 apresenta as estatísticas descritivas para as proxies de liquidez antes e após o início da atuação dos market makers. Em relação aos valores nela reportados notase que, quando observados os valores para o bid-ask-spread e para o número de negócios, houve uma elevação na liquidez dos ativos com a contratação do market maker, ao passo que, em relação ao volume, o que se viu foi uma redução da liquidez com o início da atuação desse agente de mercado.

Tabela 6

Estatísticas descritivas antes e depois do início da atuação dos market makers

\begin{tabular}{ccccc}
\hline & Mínimo & Máximo & Média & Desvio padrão \\
\hline Média do bid-ask-spread antes & 0,04 & 13,07 & 3,03 & 2,58 \\
Média do bid-ask-spread depois & 0,13 & 15,93 & 2,29 & 2,66 \\
Média do número de negócios antes & 0,00 & 4,00 & 1,41 & 0,75 \\
Média do número de negócios depois & 1,13 & 109,19 & 4,42 & 16,59 \\
Média do volume antes & 898 & 365965 & 13143,30 & 55930,96 \\
Média do volume depois & 267 & 186817 & 7610,11 & 28831,18 \\
\hline
\end{tabular}

Fonte: Dados da pesquisa (2019).

Para prosseguimento da análise faz-se necessária a realização de um teste de normalidade, a fim de identificar qual o melhor teste de comparação de médias (paramétrico ou não paramétrico). Com isso, realizou-se o teste de Kolmogorov-Sminorv, que é mais adequado para o tamanho da amostra (42 BDRs). Expressos na Tabela 7, os resultados desse teste rejeitaram a hipótese nula, em uma evidência de que os dados não seguem uma distribuição normal, sendo mais indicado para comparação de médias um teste não paramétrico. 
Tabela 7

\section{Teste de normalidade para as médias}

\begin{tabular}{cccc}
\hline & \multicolumn{3}{c}{ Kolmogorov-Smirnov } \\
& Estatística & df & Sig. \\
\hline Média do bid-ask-spread antes & 0,154 & 42 & 0,014 \\
Média do bid-ask-spread depois & 0,262 & 42 & 0,000 \\
Média do número de negócios antes & 0,173 & 42 & 0,003 \\
Média do número de negócios depois & 0,463 & 42 & 0,000 \\
Média do volume antes & 0,439 & 42 & 0,000 \\
Média do volume depois & 0,443 & 42 & 0,000 \\
\hline
\end{tabular}

Fonte: Dados da pesquisa (2019).

Por fim, na Tabela 8 são apresentados os resultados para o teste de Wilcoxon, que foi realizado com o objetivo de comparar as médias para as proxies de liquidez antes e depois do início de atuação dos market makers. Os resultados desse teste foram unânimes em apontar para a rejeição da hipótese nula, sendo uma evidência de que as médias são diferentes para os períodos antes e após a atuação do formador de mercado.

Tabela 8

Teste de Wilcoxon para a média de duas amostras pareadas

\begin{tabular}{cccc}
\hline & $\begin{array}{c}\text { Média do bid-ask-spread antes - } \\
\text { Média do bid-ask-spreaddepois }\end{array}$ & $\begin{array}{c}\text { Média do número de } \\
\text { negócios antes - } \\
\text { Média do número de } \\
\text { negócios depois }\end{array}$ & $\begin{array}{c}\text { Média do volume antes - } \\
\text { Média do volume depois }\end{array}$ \\
\hline $\mathrm{Z}$ & $-3,407$ & $-3,407$ & $-4,082$ \\
$\begin{array}{c}\text { Significância } \\
\text { Sig. (2 } \\
\text { extremidades) }\end{array}$ & 0,001 & 0,001 & 0,000 \\
\hline
\end{tabular}

Fonte: Dados da pesquisa (2019).

Os resultados apontam que os benefícios da adoção de market makers foram válidos em grande parte dos ativos analisados. Mesmo com intensidades diferentes para cada proxy estudada, o resultado supera os encontrados em Ambrozini et al. (2009) e Costa e Salles (2010) e vai ao encontro dos estudos realizados por Sanvitto (2011), Silva (2012) e Rogers e Mamede (2014) ao mostrarem que a atuação dos market makers proveram liquidez e impactaram positivamente as empresas que fizeram uso desse mecanismo.

\section{CONSIDERAÇÕES FINAIS}

A liquidez de mercado executa uma função essencial na formação de portfólios de investimentos no mercado acionário. Por essa razão, definiu-se como objetivo do presente estudo apurar o efeito da introdução dos market makers no aumento da liquidez das Brazilian Depositary Receipts (BDRs) - valores mobiliários emitidos no Brasil e que possuem como lastro ativos, geralmente ações, emitidos no exterior. Para tanto, foi aplicada a metodologia estatística do teste de quebra estrutural proposto por Chow (1960) em 42 BDRs que compuseram a amostra do estudo. A função do teste de Chow foi examinar se houve mudanças abruptas nas séries temporais das proxies de liquidez (bid-ask-spread, número de negócios e volume) em cada uma das BDRs, após a contratação dos market makers.

Para a realização do teste de Chow, as séries temporais de cada proxy foram divididas em três amostras. A amostra I foi composta por dados anteriores à contratação dos market makers, a 
amostra II foi composta por dados posteriores à contratação e a amostra III foi formada por todos os dados das proxies das 42 BDRs estudadas. Para que fique demostrado que a atuação dos market makers impactaram na liquidez dos ativos é preciso que haja diferenças estatisticamente significativas entre a SQR da amostra III com a SQR das amostras I e II. Após o resultado, rejeitase ou não a hipótese nula com base no p-valor e nos níveis de significância de 1\%, 5\% e 10\%.

A partir da análise do p-valor gerado em cada teste, foi possível observar que, conforme esperado, na maioria das BDRs estudadas a introdução de market makers ocasionou mudanças nas três proxies de liquidez. Os resultados encontrados na análise do teste de Chow a um nível de confiança de 99\% apontaram que, após a contratação dos market makers, 29 BDRs, ou seja, 69\% das BDRs estudadas, tiveram mudanças abruptas e estatisticamente significativas no bid-askspread, $23(55 \%)$ apresentaram mudanças abruptas no número de negócios e 22 (52\%) apresentaram mudanças no volume. Ao flexibilizar o nível de confiança para 95\%, 32 BDRs (76\%) apresentaram mudanças abruptas no bid-ask-spread, 29 ou $69 \%$ apresentaram mudanças no número de negócios e 29 , ou seja, $69 \%$ tiveram mudanças no volume de negociações. Ao nível de confiança de $90 \%$, os resultados encontrados foram de 35 BDRs $(83 \%)$ apresentando mudanças no bid-ask-spread, 32 BDRs (76\%) apresentando mudanças no número de negócios e 30 (71\%) apresentando mudanças abruptas e estatisticamente significativas no volume.

Nesse sentido, foi possível rejeitar a hipótese nula de que não houve quebra estrutural abrupta nas séries temporais das proxies de liquidez em grande parte das BDRs presentes na amostra estudada. Esse resultado fornece, portanto, fortes evidências sobre a influência dos market makers na liquidez de mercado das BDRs, mostrando que a contratação desses formadores de mercado pode aumentar a liquidez e contribuir de maneira significativa com as negociações desses papéis, facilitando sua comercialização por parte dos investidores. Além disso, esse resultado corrobora os achados de outras pesquisas que abordam o impacto na liquidez proveniente da introdução de market makers, como os trabalhos de Ambrozini et al. (2009), Costa e Salles (2010), Sanvitto (2011), Silva (2012), Perlin (2013) e Rogers e Mamede (2014).

Uma das restrições deste trabalho é apresentar apenas se houve ou não aumento de liquidez depois da contratação dos market makers, mas não informa o quanto a liquidez foi impactada. Apesar da limitação, os resultados respondem à questão de pesquisa e contribuem para o estudo sobre a liquidez de mercado e sobre como os market makers contribuem para a sua elevação. Além disso, este estudo abre caminho para novas possibilidades de pesquisa, como verificar o grau de influência dos market makers na liquidez, bem como a realização de uma análise focada nos market makers contratados, a fim de mostrar quais foram mais eficientes na promoção da liquidez de mercado nas empresas que os contrataram. Sugere-se ainda, a realização de uma avaliação mais criteriosa sobre como a atuação dos market makers provoca retornos anormais ou reduz o risco para o investidor a partir das mudanças ocasionadas na liquidez. Claramente tais questões seriam interessantes de serem respondidas futuramente, de maneira a contribuir ainda mais com o entendimento dos fatores que interferem na liquidez de mercado e as consequências dessa interferência.

\section{REFERÊNCIAS}

Ambrozini, M. A., Gaio, L. E., Bonacim, C. A. G., \& Cicconi, E. G. (2009). Impacto dos formadores de mercado sobre a Liquidez das Ações Negociadas na Bolsa de Valores de São Paulo. Contabilidade Vista \& Revista, 20(3), 15-38.

Amihud, Y., \& Mendelson, H. (1986). Asset pricing and the bid-ask spread. Journal of financial Economics, 17(2), 223-249.

Amihud, Y., \& Mendelson, H. (1991). Liquidity, asset prices and financial policy. Financial Analysts Journal, 47(6), 56-66. 
Amihud, Y., \& Mendelson, H. (2008). Liquidity, the value of the firm, and corporate finance. Journal of Applied Corporate Finance, 20(2), 32-45.

Amihud, Y., \& Mendelson, H. (1987). Trading mechanisms and stock returns: An empirical investigation. The Journal of Finance, 42(3), 533-553.

Banz, R. W. (1981). The relationship between return and market value of common stocks. Journal of Financial Economics, 9(1), 3-18.

Box, G. E., \& Jenkis, G. M. (1970). Time series analysis for casting and control. San Francisco, Holden Day (44), 575.

Brasil Bolsa Balcão (B3). BDRs - Brazilian Depositary Receipts. Recuperado em 11 dezembro, 2017, de http://www.bmfbovespa.com.br/pt_br/listagem/bdrs-brazilian-depositaryreceipts/.

Chordia, T., Subrahmanyam, A., \& Anshuman, V. R. (2001). Trading activity and expected stock returns. Journal of financial Economics, 59(1), 3-32.

Chow, G. C. (1960). Tests of equality between sets of coefficients in two linear regressions. Econometrica: Journal of the Econometric Society, 28(6), 591-605.

Clark-Joseph, A. D., Ye, M., \& Zi, C. (2017). Designated market makers still matter: Evidence from two natural experiments. Journal of Financial Economics, 126(3), 652-667.

Copeland, T. E., \& Galai, D. (1983). Information effects on the bid-ask spread. The Journal of Finance, 38(5), 1457-1469.

Correia, L. F., Amaral, H. F., \& Bressan, A. A. (2008). O efeito da liquidez sobre a rentabilidade de mercado das ações negociadas no mercado acionário brasileiro. BASE-Revista de Administração e Contabilidade da Unisinos, 5(2), 109-119.

Costa, A. C., \& Salles, A. A. (2010, Setembro). Um Exame da Influência do Formador de Mercado no Risco de Liquidez de Ações Negociadas. Anais do Encontro Nacional de Engenharia de Produção, São Carlos, SP, Brasil, 30

Demsetz, H. (1968). The cost of transacting. The quarterly journal of economics, 82(1), 33-53.

Fama, E. F. (1970). Efficient capital markets: A review of theory and empirical work. The journal of Finance, 25(2), 383-417.

Fama, E. F. (1991). Efficient capital markets: II. The journal of finance, 46(5), 1575-1617.

Garman, M. B. (1976). Market microstructure. Journal of Financial Economics, 3(3), 257-275.

Glosten, L. R. (1989). Insider trading, liquidity, and the role of the monopolist specialist. Journal of Business, 62(2), 211-235.

Glosten, L. R., \& Milgrom, P. R. (1985). Bid, ask and transaction prices in a specialist market with heterogeneously informed traders. Journal of financial economics, 14(1), 71-100. 
Jun, S. G., Marathe, A., \& Shawky, H. A. (2003). Liquidity and stock returns in emerging equity markets. Emerging Markets Review, 4(1), 1-24.

Kyle, A. S. (1985). Continuous auctions and insider trading. Econometrica: Journal of the Econometric Society, 53(6), 1315-1335.

Machado, M. A. V., \& Medeiros, O. R. (2011). Modelos de precificação de ativos e o efeito liquidez: evidências empíricas no mercado acionário brasileiro. Revista Brasileira de Finanças, 9(3).

Madhavan, A. (2000). Market microstructure: A survey. Journal of Financial Markets, 3(3), 205258.

Markowitz, H. (1952). Portfolio selection. The journal of finance, 7(1), 77-91.

Martins, G. A., \& Theóphilo, C. R. (2007). Metodologia da investigação científica para Ciências Sociais Aplicadas. São Paulo, Brasil: Atlas.

Morais, I. A., \& Portugal, M. S. (1999). Modelagem e previsão de volatilidade determinística e estocástica para a série do Ibovespa. Journal of Economic Literature, 29(3), 303-341.

Neves, L. C. (2007). Estudo Empírico sobre o Comportamento do retorno e da liquidez média das ações no Mercado Acionário Brasileiro das Empresas que emitiram ADRs na NYSE e das que aderiram ao Novo Mercado. Dissertação de Mestrado, Universidade Federal de Uberlândia, Uberlândia, MG, Brasil.

O’Hara, M. (1995). Market Microstructure Theory. (1a. ed.). Cambridge: Blackwell Publishers.

Pereira, A. J., Neto. (2006). Testes de estrutura ótima de capital em empresas brasileiras: o efeito de liquidez, desempenho do mercado acionário e assimetria de informação nas decisões de financiamento. Tese de Doutorado, Fundação Getúlio Vargas, São Paulo, SP, Brasil.

Perlin, M. (2013). Os efeitos da introdução de agentes de liquidez no mercado acionário brasileiro. Revista Brasileira de Finanças, 11(2), 281-304.

Perotti, P., \& Rindi, B. (2010). Market makers as information providers: The natural experiment of STAR. Journal of Empirical Finance, 17(5), 895-917.

Radner, R. (1979). Rational expectations equilibrium: Generic existence and the information revealed by prices. Econometrica: Journal of the Econometric Society, 655-678.

Rogers, P., \& Mamede, S. D. P. N. (2014). Ineficiência informacional e/ou valor da liquidez? Estudo da atuação dos formadores de mercado no Brasil. Anais do Encontro Brasileiro de Finanças. Recife, PE, Brasil, 14.

Sanvitto, M. A. (2011). Formadores de mercado como agentes provedores de liquidez e de valor: um confronto entre teoria e realidade. Monografia, Universidade Federal do Rio Grande do Sul, Porto Alegre, RS, Brasil.

Silva, V. A. D. (2012). Uma análise sobre a liquidez e a volatilidade das ações de empresas que 
contrataram formadores de mercado. Monografia, Universidade Federal de Santa Catarina, Florianópolis, SC, Brasil.

Siqueira, L. S., Amaral, H. F., \& Correia, L. F. (2017). The effect of asymmetric information risk on returns of stocks traded on the BM\&FBOVESPA. Revista Contabilidade \& Finanças, 28(75), 425-444.

Stoll, H. R. (1978). The supply of dealer services in securities markets. The Journal of Finance, 33(4), 1133-1151.

Venkataraman, K., \& Waisburd, A. C. (2007). The value of the designated market maker. Journal of Financial and Quantitative Analysis, 42(3), 735-758.

Vieira, K. M., \& Milach, F. T. (2008). Liquidez/Iliquidez no Mercado Brasileiro: Comportamento no Período 1995-2005 e suas Relações com o Retorno. BASE-Revista de Administração e Contabilidade da Unisinos, 5(3), 5-16. 